\title{
PRIMAL SUBMODULES OF MULTIPLICATION MODULES
}

\author{
S. EBRAHIMI ATANI AND A. YOUSEFIAN DARANI
}

\begin{abstract}
Let $R$ be a commutative ring with non-zero identity. In this paper we characterize primal submodules of multiplication modules.
\end{abstract}

\section{Introduction}

Throughout this paper all ring are considered commutative rings with nonzero identity, and all modules are unitary. Let $M$ be an $R$-module. An $R$-module $M$ is called multiplication if for any submodule $N$ of $M$ we have $N=I M$, where $I$ is an ideal of $R$. In this case we can take $I=\left(N:_{R} M\right)$. If $P$ is a maximal ideal of $R$, then $T_{P}(M)=\{m \in M:(1-p) m=0$ for some $p \in P\}$ is a submodule of $M$. We say that $M$ is $P$-cyclic provided there exists $q \in P$ and $m \in M$ such that $(1-q) M \subseteq R m$. So $M$ is a multiplication module if and only if for every maximal ideal $P$ of $R$ either $M=T_{P}(M)$ or $M$ is $P$-cyclic (see [2, Theorem 1.2]).

The concept of primal ideals in a commutative ring was introduced by L. Fuchs in [4]. Let $R$ be a commutative ring and let $I$ be an ideal of $R$. An element $a \in R$ is called prime to $I$ if $r a \in I(r \in R)$ implies $r \in I$, so $\left(I:_{R} r\right)=I$. Denote by $S(I)$ the set of all elements of $R$ that are not prime to $I$. A proper ideal $I$ of $R$ is said to be primal if $S(I)$ forms an ideal of $R$; this ideal is always a prime ideal, called the adjoint ideal $P$ of $I$. In this case we also say that $I$ is a $P$-primal ideal.

The concept primal submodules have been introduced by D. Dauns in [1]. Also, this class of submodules is studied extensively in [3]. Let $R$ be a commutative ring, $M$ an $R$-module and $N$ an $R$-submodule of $M$. An element $r \in R$ is called prime to $N$ if $r m \in N(m \in M)$ implies that $m \in N$. In this case, $(N: M r)=\{m \in M: r m \in N\}=N$. Denote by $S(N)$ the set of all elements of $R$ that are not prime to $N$. A proper submodule $N$ of $M$ is said to be primal if $S(N)$ forms an ideal of $R$; this ideal is called the adjoint ideal $P$ of $N$. In this case we also say that $N$ is a $P$-primal submodule of $M$ (see [1], [3]).

A proper submodule $N$ of a module $M$ over a $\operatorname{ring} R$ is said to be prime ( $P$-prime) if $r m \in N$ for $r \in R$ and $m \in M$ implies that either $m \in N$ or $r \in\left(N:_{R} M\right)=P$. A proper submodule $N$ of a module $M$ over a ring $R$ is said to be primary if $r m \in N$ for $r \in R$ and $m \in M$ implies that either $m \in N$ or $r^{s} \in\left(N:_{R} M\right)$ for some $s[6]$.

In [2], Z. A. El-Bast and P. F. Smith proved that if $M$ is faithful multiplication and $P$ a prime ideal of $R$ such that $M \neq P M$ then $P M$ is a prime submodule of $M$. In this paper, we first

Received February 20, 2008.

2000 Mathematics Subject Classification. 13A05, 13F05.

Key words and phrases. Primal, prime, multiplication. 
give an example (Example 2.1) in which we show that all submodules of the $Z$-module $E(p)$ are primal. This example also show that a primal submodule need not be neither prime nor primary. Then we prove that if $M$ is a faithful finitely generated multiplication and $I$ a primal ideal of $R$ such that $M \neq I M$ then $I M$ is a primal submodule of $M$. Also, a number of results concerning primal submodules of a multiplication are given (see Section 2).

\section{The results}

The class of primal submodules is a large class. For example, all primary submodules and irreducible submodules are primal [3,4]. Therefore, the structure of primal submodules is worthy of study. Our starting point is the following example:

Example 2.1. Let $p$ be a fixed prime integer and $\mathbb{N}_{0}=\mathbb{Z}^{+} \cup\{0\}$. Then $E(p)=\{\alpha \in Q / \mathbb{Z}$ : $\alpha=r / p^{n}+\mathbb{Z}$ for some $r \in \mathbb{Z}$ and $\left.n \in \mathbb{N}_{0}\right\}$ is a non-zero submodule of the $\mathbb{Z}$-module $Q / \mathbb{Z}$. For each $t \in \mathbb{N}_{0}$, set $G_{t}=\left\{\alpha \in Q / \mathbb{Z}: \alpha=r / p^{t}+\mathbb{Z}\right.$ for some $\left.r \in \mathbb{Z}\right\}$. $G_{t}$ is a cyclic submodule of $E(p)$ generated by $1 / p^{t}+\mathbb{Z}$ for each $t \in \mathbb{N}_{0}$. Each non-zero proper submodule $E(p)$ is equal to $G_{i}$ for some $i \in \mathbb{N}_{0}$. Also, $\left(G_{t}: Z E(p)\right)=0$ for every $t \in \mathbb{N}_{0}[6]$. However, no $G_{t}$ is primary submodule of $E(p)$, for if $p^{k} \notin\left(G_{t}:_{Z} E(p)\right)=0$ for all $k \in \mathbb{N}_{0}$ and $1 / p^{i+t}+Z \notin G_{t}$, but $p^{i}\left(1 / p^{i+t}+Z\right)=$ $1 / p^{t}+Z \in G_{t}$.

By [1, Example 1.14], $G_{0}$ is a primal submodule of $E(p)$. We claim that, for every $t \in \mathbb{N}_{0}$, $G_{t}$ is a $p \mathbb{Z}$-primal submodule of $E(p)$. If $p k \in p \mathbb{Z}$, we have $p k \alpha_{t+1}=k \alpha_{t} \in G_{t}$ but $\alpha_{t+1} \notin G_{t}$. So $p k$ is not prime to $G_{t}$. Now assume that $n \in \mathbb{Z}$ is not prime to $G_{t}$. Then there exists a positive integer $k>t$ with $0 \neq a_{k}=r / p^{k}+\mathbb{Z} \in E(p)-G_{t}$ such that $n a_{k} \in G_{t}$. So $n\left(r / p^{k}+\mathbb{Z}\right)=$ $r^{\prime} / p^{t}+\mathbb{Z}$ for some $r^{\prime} \in \mathbb{Z}$. We may assume that $r$ and $p$ are coprime. So there exists $m \in \mathbb{Z}$ with $r n-r^{\prime} p^{k-t}=p^{k} m$. It follows that $p \mid r n$ and hence $p \mid n$. Thus, $p \mathbb{Z}$ consists exactly of the set of elements of $\mathbb{Z}$ which are not prime to $G_{t}$. Hence $G_{t}$ is a $p \mathbb{Z}$-primal submodule of $E(p)$. Hence, a primal submodule need not be a primary (so prime) submodule.

Proposition 2.2. Let I be a $P$-primal ideal of a commutative ring $R$ and $M$ a faithful multiplication R-module. Let $a \in R, x \in M$ satisfy ax $\in I M$. Then $a \in P$ or $x \in I M$.

Proof. Suppose that $a \notin P$ (so $a$ is prime to $I$ ); we show that $x \in I M$. Set $J=\{r \in R: r x \in$ $I M$ 3. Suppose $J \neq R$. Then $J \subseteq Q$ for some maximal ideal $Q$ of $R$. If $x \in T_{Q}(M)$, then there is an element $q \in Q$ such that $(1-q) x=0 \in I M$, so $1-q \in J \subseteq Q$ which is a contradiction. So we may assume that $M$ is $Q$-cyclic. Then $(1-q) M \subseteq R m$ for some $q \in Q$ and $m \in M$, so there exists $s \in R$ such that $(1-q) x=s m$; hence $(1-q) a x=a s m=b m$ for some $b \in I$ since $(1-q) I M \subseteq I m$. Therefore, $a s-b \in\left(0:_{R} m\right)$. Since $M$ is faithful and $(1-q)\left(0:_{R} m\right) M \subseteq R\left(0:_{R} m\right) m=0$, we must have $(1-q)\left(0:_{R} m\right)=0$; hence $(a s-b)(1-q)=0$. It follows that $(1-q) b=(1-q) a s \in I$. Since $a$ is prime to $I$, we get $(1-q) s \in I$; hence $s \in I$ since $1-q \notin P$; thus $(1-q) x=s m \in I M$. Therefore, $(1-q) \in J \subseteq Q$, a contradiction. It follows that $J=R$ and $x \in I M$, as needed.

Theorem 2.3. Let I be a $P$-primal ideal of a commutative ring $R$ and $M$ a faithful finitely generated multiplication $R$-module such that $I M \neq M$. Then IM is a P-primal submodule of M. 
Proof. It suffices to show that the set of elements of $R$ that are not prime to $I M$ is just $P$. Suppose that $s$ is an element of $R$ such that it is not prime to $I M$. So there exists $m \in M-I M$ such that $s m \in I M$. It then follows from Proposition 2.2 that $s \in P$. Conversely, assume that $s \in P$. Then $s t \in I$ for some $t \in R-I$. Since $M$ is faithful finitely generated multiplication, we must have $t M \nsubseteq I M$ (because $(I M: M)=I$ ); hence $t m \notin I M$ for some $m \in M-I M$.. As $t s m \in I M$ with $t m \notin I M$, we get $s$ is not prime to $I M$, and the proof is complete.

Lemma 2.4. Let I be an ideal of a commutative ring $R, M$ an $R$-module and $N$ a proper $R$-submodule of $M$ such that $I \subseteq(0: R M)$. Then $N$ is a primal $R$-submodule of $M$ if and only if $N$ is a primal submodule of $M$ as an R/I-module.

Proof. The proof is straightforward.

Theorem 2.5. The following statements are equivalent for a proper submodule $N$ of a finitely generated multiplication over a commutative ring $R$ :

(i) $N$ is a primal submodule of $M$.

(ii) $I=\left(N:_{R} M\right)$ is a primal ideal of $R$.

(iii) $N=Q M$ for some primal ideal $Q$ of $R$ with $\operatorname{Ann}(M) \subseteq Q$.

Proof. $(i) \rightarrow(i i)$. Assume that $N$ is a $P$-primal submodule of $M$; we show that $I$ is a $P$ primal ideal of $R$. It suffices to show that the set of elements of $R$ that are not prime to $I$ is just $P$. Suppose that $s$ is an element of $R$ such that is not prime to $I$, so there is an element $t \notin I$ such that $s t \in I$; hence there exist $m \in M-N$ with $t m \notin N$ and $s(t m) \in N$. Therefore, $s$ is not prime to $N$, so $s \in P$. Conversely, assume that $s \in P$. Then there exists $m \in M-N$ such that $s m \in N$. Now we show that $s$ is not prime to $I$. Suppose not. Then $\left(I:_{R} s\right)=I$. An inspection will show that $\left(I:_{R} s\right) M=\left(N:_{M} s\right)=I M=N$ [4]; hence $s \in N$, which is a contradiction. ( $\left.i i\right) \rightarrow$ (iii) is clear.

(iii) $\rightarrow($ i $)$. Since $N=Q M \neq M$ and as an $R /(0: R M)$-module, $N$ is primal by Theorem 2.3, so is primal as an $R$-submodule of $M$ by Lemma 2.4.

Definition 2.6. Let $R$ be a commutative ring with identity. $R$ is called a $P$-ring if and only if every ideal in $R$ is product of primal ideals.

Definition 2.7. Let $M$ be a module over a commutative ring $R$. $M$ is called $P$-module if every proper submodule $N$ of $M$ either is primal or has a primal factorization $N=P_{1} P_{2} \cdots P_{n} N^{*}$, where $P_{1}, \ldots, P_{n}$ are primal ideals of $R$ and $N^{*}$ is a primal submodule in $M$.

Theorem 2.8. Let $M$ be a faithful finitely generated multiplication module over a $P$-ring $R$. Then $M$ is a P-module.

Proof. Let $N$ be a proper submodule of $M$. Then $N=I M$ for some ideal $I$ of $R$. Since $R$ is $P$-ring, we must have $I=P_{1} P_{2} \cdots P_{k}$ and so $N=P_{1} P_{2} \cdots P_{k} M$ where $P_{1}, P_{2}, \ldots, P_{k}$ are primal ideals of $R$. Since $N$ is proper, we must have $P_{i} M \neq M$ for some $1 \leq i \leq k$; hence $P_{i} M$ is a primal submodule of $M$ by Proposition 2.2, as required. 
Recall that a ring $R$ is called a $Z P I$ ring if every ideal of $R$ can be wrritten as a product of prime ideals of $R$. Also, we recall that, by [1, Proposition 1.9], over a commutative ring $R$, every prime submodule is primal; hence every $Z P I$ ring is a $P$-ring. Then we have the following corollary:

Corollary 2.9. If $R$ is a ZPI ring (resp. Dedekind domain) and $M$ a faithful finitely generated multiplication $R$-module, then $M$ is a $P$-module.

Theorem 2.10. Let $M$ be a finitely generated faithful multiplication module over a commutative ring $R$. If $M$ is a $P$-module, then $R$ is $P$-ring.

Proof. Let $I$ be a proper ideal of $R$. Then [2, Theorem 3.1] gives $I M \neq M$, so $I M=$ $P_{1} P_{2} \cdots P_{n} N^{*}$ where $P_{1}, P_{2}, \ldots, P_{n}$ are primal ideals of $R$ and $N^{*}$ is a primal submodule of $M$. We can write $N^{*}=\left(N^{*}:_{R} M\right) M$ where $\left(N^{*}:_{R} M\right)$ is primal by Theorem 2.5. Therefore, $I M=P_{1} P_{2} \cdots P_{n}\left(N^{*}:_{R} M\right) M$, so $I=P_{1} P_{2} \cdots P_{n}\left(N^{*}:_{R} M\right)$ by [2, Theorem 3.1]. Thus $M$ is a $P$-ring.

\section{References}

[1] J. Dauns, Primal modules. Comm. Algebra 25 (1997), 2409-2435.

[2] Z. A. El-Bast and P. F. Smith, Multiplication modules. Comm. Algebra 16 (1988), 755-779.

[3] S. Ebrahimi Atani and A. Yousefian Darani, Some remarks on primal submodules. to appear in Sarajevo Journal of Mathematics 17(2008), 181-190.

[4] S. Ebrahimi Atani and A. Yousefian Darani, Notes on the primal submodules, Chiang Mai J. Sci. 53(2008), 399-410.

[5] L. Fuchs, On primal ideals. Proc. Amer. Math. Soc. 1 (1950) 1-6.

[6] C. P. Lu, Spectra of modules. Comm. Algebra, 23 (1995), 3741-3752.

Department of Mathematics, University of Guilan, P.O. Box 1914, Rasht Iran.

E-mail: ebrahimi@guilan.ac.ir

Department of Mathematics, University of Mohaghegh Ardabili, P.O. Box 179, Ardabil, Iran.

E-mail:yousefian@uma.ac.ir 